\title{
Features of acoustic resonance in iron borate
}

\author{
K.M.Skibinsky*, M.B.Strugatsky*, S.V.Yagypov*, \\ A.I.Yevstafyev ${ }^{* * *}$, V.L.Preobrazensky ${ }^{* *}$, V.N.Berzansky \\ Joint European Laboratory LICS/LEMAC: "Taurida National University, \\ 4 Vernadsky Ave., 95007 Simferopol, Ukraine \\ *: IEMN CNRS 8520, Ecole Centrale de Lille, BP 48, \\ 59651 Villeneuve d'Ascq, France
}

Received January 10, 2014

\begin{abstract}
Acoustic resonance in a weak-ferromagnetic iron borate monocrystal was investigated. It was shown that observed field dependence of positions and magnitudes of the resonance peaks can be explained within framework of the developed theory of magnetic birefringence of sound in the inhomogeneous magnetized sample.
\end{abstract}

\begin{abstract}
Исследован акустический резонанс в слабоферромагнитном монокристалле бората железа. Показано, что наблюдающаяся полевая зависимость положений и величин резонансных пиков может быть объяснена в рамках развитой теории магнитного двупреломления звука в неоднородно намагниченном образце.
\end{abstract}

Особливості акустичного резонансу в бораті заліза. К.М.Скібінський, М.Б.Стругацький, С.В.Ягупов, А.І.Євстаф'єв, В.Л.Преображенський, В.Н.Бержанський.

Досліджено акустичний резонанс в слабкоферомагнітному монокристалі бората заліза. Показано, що спостережена польова залежність положень і величин резонансних піків може бути пояснена у рамках розвиненої теорії магнітного двозаломлення звуку в неоднорідно намагніченому зразку.

\section{Introduction}

Acoustic resonance in free suspended thin basal plate $\left(\perp C_{3}\right)$ of antiferromagnet $\mathrm{FeBO}_{3}$ crystal was investigated in the work [1]. Sound was exited by magnetic field of radio frequency acting in the plane of the sample. Dependence of sound resonance frequency $\omega_{\text {res }}$ on static magnetic field applied in the basal plane was found. In this case it was exited the first-order resonance, corresponding to the first harmonic of standing acoustic waves. Multimoding observed in the weak magnetic fields was associated with possible nonuniform strains in the sample and/or the presence of domains [1]. This multimoding appears in the initial part of the curve $\omega_{\text {res }}(H)$.

In the work [2] several branches $\omega_{\text {res }}(H)$ corresponding to the acoustic Fabry-Perot resonances of different orders were ob- served in synthesized by us thin $(\sim 140 \mu \mathrm{m})$ highly perfected basal plate of iron borate at temperature $77 \mathrm{~K}$ (Fig. 1, circles). In this case the sound was exited and registered by piezoelectric transducers attached to natural basal faces of the crystal. Transverse acoustic waves propagating along three-fold axis were produced in the crystal. Many-fold rereflections from the crystalpiezotransducer boundaries under size resonance conditions led to a sharp increase of the wave amplitude registered by the piezoreceiver. All observed acoustic resonances conventionally could be divided into two groups: in the first group the shift of resonances under magnetic field change (mainly in the weak fields region) was much stronger (Fig. 1, open circles) than in the second one (Fig. 1, filled circles). We named the acoustic wave modes producing strongly 


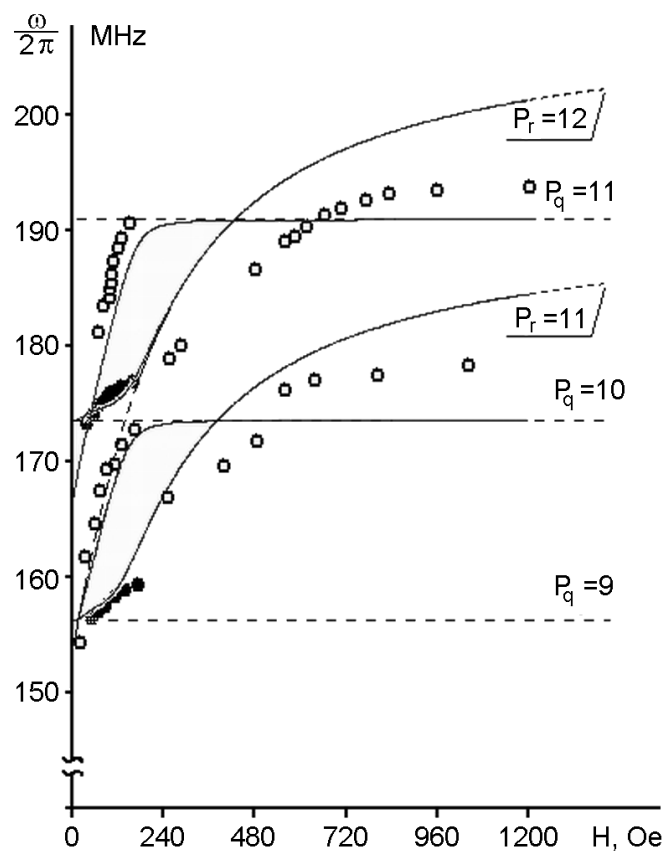

Fig. 1. Field dependence of resonance frequencies: circles - the experiment [2] (open circles - strongly-magnetic modes; filled circles — weakly-magnetic modes); lines - the theory [5] (dotted lines - pure magnetic and nonmagnetic modes; solid lines - basal-anisotropic nonuniform model).

shifted resonances as the strongly-magnetic modes. When the shift is weak we speak about the weakly-magnetic modes.

The present work is devoted to experimental and theoretical investigations of the features of acoustic resonance in iron borate.

\section{Electromagnetic excitation of the acoustic resonances in iron borate}

We experimentally observed a characteristic "overflow" of the resonances from one to another (Fig. 2) at electromagnetic excitation of the acoustic resonances in a disk with the diameter $3 \mathrm{~mm}$ and thickness $0.1 \mathrm{~mm}$, made from monocrystal basal plate $\mathrm{FeBO}_{3}$ grown by us. Note, that such behavior does not correspond to the resonances that could be generated by pure magnetic and nonmagnetic modes: "magnetic" resonance should shift over the entire range of the fields while "nonmagnetic" one is not shifted. The sample was placed in an organic glass frame with two perpendicular coils wound around it. Geometry of the experiment is presented in Fig. 3, where $H$ is the external bias magnetic field, $h p$ is the pumping coil field, CA is the detection coil axis which is perpendicular to the $h_{p}$. The sample could freely oscillate inside the frame. As coils were aligned at 90 degrees, their mutual induction was close to zero. In this case if a harmonic signal was applied to the pumping coil, it was not visible at the receiving coil. However if the pumping frequency was close to the frequency of magnetoelastic mode of the resonator in selected bias magnetic field, the sample oscillated and thus generated signal in the detection coil by projection of vibrating magnetic moment. The power of input signal was $30 \mathrm{dBm}$ and the temperature was $223 \mathrm{~K}$. External bias magnetic field $H$ was applied

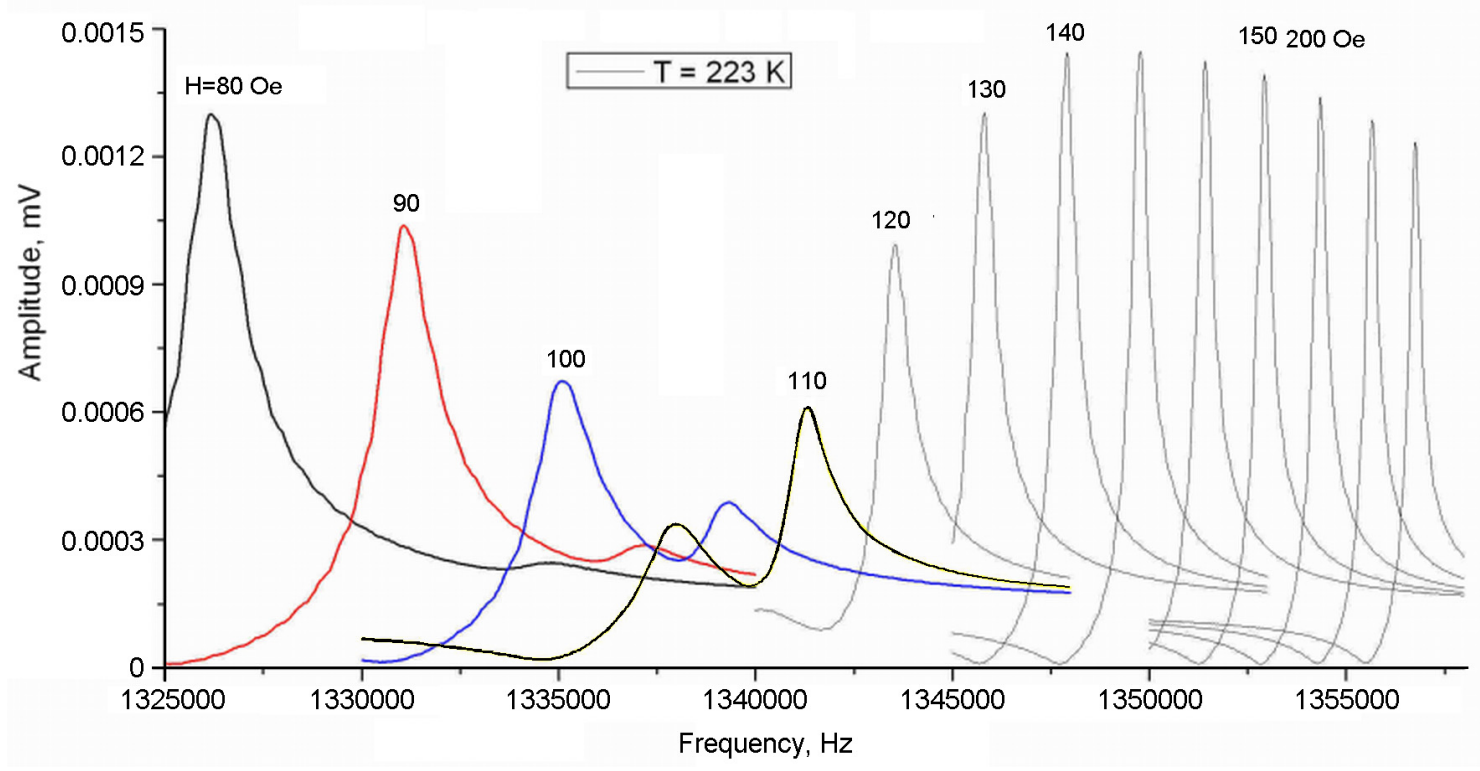

Fig. 2. Frequencies dependence of amplitude: the experiment. 
in the basal crystalline plane of the sample at an angle of about 80 degrees with respect to the alternating magnetic field of the pumping coil. Small declining from perpendicular geometry was used to provide induction in the detecting coil from ferromagnetic moment oscillations.

Fig. 2 demonstrates a series of curves registered at the detection coil that show crystal oscillation amplitude dependence on pumping frequency in the different bias magnetic fields $H=80 \div 200$ Oe.

\section{Calculation of the resonance curves}

It proved possible to analyze the experimental dependences $\omega_{\text {res }}(H)$ [2] on the basis of our models [3 - 5] that describe the features of magnetic birefringence of sound in this crystal - acoustic analog of the well known Cotton-Mouton optical effect. Theory of magnetic linear birefringence of transverse sound in trigonal easy-plane antiferromagnet with the weak ferromagnetism was developed in the work [6]. According to [6], a linearly polarized acoustic wave, entering the crystal, is transformed into two modes in the crystal: nonmagnetic and magnetic ones, polarized in mutually orthogonal planes. The phase velocity of the magnetic mode depends on magnetic field applied in the basal plane. We are talking about the lower quasiphonon branch of magnetoelastic excitations. The upper quasimagnon branch, which corresponds to the frequencies of the ferromagnetic resonance, is not excited. In this case magnetic oscillations follow the elastic ones quasistaticaly. So, each resonance would have to correspond only to nonmagnetic or magnetic mode of the acoustic wave in the crystal. But purely nonmagnetic resonances are not observed in the experiments [2] at all. To interpret the weaklymagnetic resonances [2] we had to take into account the effect of experimental boundary conditions [5] caused by mechanical contact between the piezotransducers and the basal faces of the crystal. Because of strong magnetoelastic coupling in iron borate [3], such boundary conditions should affect significantly the magnetic state of the crystal, thus changing the parameters of the sound wave in the sample. We could describe the strongly-magnetic and weakly-magnetic modes [2] in the frame of the following model [5]. Deformations in the crystal, caused by mechanical boundary conditions, induce through magnetoelastic coupling the uniaxial magnetic anisotropy in the basal

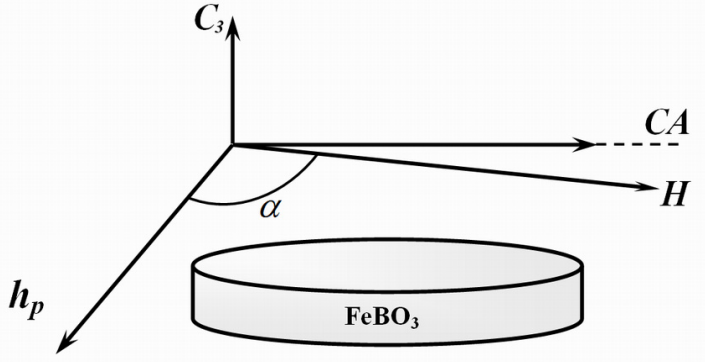

Fig. 3. Geometry of the experiment. $H$ is the bias magnetic field, $h_{p}$ is the pumping coil field, $C_{3}$ is the threefold axis of the crystal, $\mathrm{CA}$ is the detection coil axis which is perpendicular to $h_{p}$.

plane. Lately an existence of induced uniaxial anisotropy was confirmed in the magneto-acoustic experiments on hematite crystals [8], isostructural to iron borate. Magnetoelastic coupling is determined by magnetoelastic contribution $\Delta C$ to effective elastic module of the crystal. Generalizing results [6,7], we obtained the expression $\Delta C(H)$ for basal-anisotropic case [3]:

$-\frac{\Delta C=}{C_{44} H_{E} H_{m e 2}} \frac{\text { (1) }}{2 H_{E} H_{m e 1}+H \cdot\left[H \sin (\alpha-\theta)+H_{D}\right] \cdot \sin (\alpha-\theta)+4 a\left(H_{E} / M_{0}\right) \cos 2 \theta}$,

where $C_{44}$ is the elastic module; $H_{E}$ is the exchange field; $H_{D}$ is the Dzyaloshinsky field; $H_{m e 1}$ and $H_{m e 2}$ are the first and the second magnetoelastic fields [6]; $\alpha$ is the angle between the easy axis (EA) and the magnetic field; $\theta$ is the angle between the EA and the antiferromagnetic vector [3]; $M_{0}$ is the sublattice magnetization; $a$ is the constant of the induced in the basal plane uniaxial magnetic anisotropy.

For adequate description of the experimental dependences [2] we assumed also that the deformations are nonuniform along $C_{3}$ axis of the crystal: they decrease from the surface to the sample's center [5]. Under these conditions the induced uniaxial magnetic anisotropy will be nonuniform in magnitude as well: maximal on the basal surface and minimal in the center of the crystal. For simplicity we took a linear law of its decrease [3]. In this case the magnetic field applied in the basal plane and not parallel to the easy magnetization axis will result in spatially nonuniform distribution of magnetization along $C_{3}$. Directions of polarization of the normal acoustic modes will change smoothly along $C_{3}$ axis that should 
lead to admixture of the modes to one another. So, in the basal-anisotropic nonuniform case it is necessary to consider the hybrid modes instead of pure nonmagnetic and magnetic ones. These modes may be apparently identified as the above-mentioned weakly- and strongly-magnetic modes. Resonances corresponding to them should shift differently when magnetic field changes. For calculation of the dependences $\omega_{\text {res }}(H)$ in the nonuniform case we determined frequency and field dependence of the wave amplitude $A(\omega, H)$. For this purpose we used the Jones matrix method [3, 4] known from optics. One may imagine the crystal plate divided into $n$ layers parallel to the basal plane with the uniform anisotropy in each layer. Such layers are assumed to be uniformly magnetized. In this case association between the incident and traversed waves is given by

$$
\left(\begin{array}{c}
U_{r}^{\text {out }} \\
U_{q}^{\text {out }}
\end{array}\right)_{N=1,3,5}=\sum_{N=1} \ldots \tau_{N}\left(\prod_{m}^{n} T_{m}\right)^{N}\left(\begin{array}{c}
U_{r}^{\text {in }} \\
U_{q}^{\text {in }}
\end{array}\right),
$$

where $\mathbf{r}$ and $\mathbf{q}$ are the polarization vectors of the magnetic and nonmagnetic modes on the crystal surface; $N$ is the multiplicity of the wave transmissions through the crystal before emerging from it; $\tau_{N}=(1-\rho)^{2} \rho^{N-1}$ is the effective transmission factor; $\rho$ is the reflection factor for the boundary crystalpiezotransducer. In (2) the Jones matrix $T_{m}$ for the $m$-th layer is determined by the expression [3]

$$
\begin{gathered}
T_{m}= \\
=\left(\begin{array}{cc}
\exp \left(-i k_{r m} d / n\right) & 0 \\
0 & \exp \left(-i k_{q} d / n\right)
\end{array}\right) \cdot\left(\begin{array}{cc}
\cos \Delta \gamma_{m} & -\sin \Delta \gamma_{m} \\
-\sin \Delta \gamma_{m} & \cos \Delta \gamma_{m}
\end{array}\right) .
\end{gathered}
$$

Here $k_{q}$ is the wave vector of the nonmagnetic mode; $k_{r m}$ is the wave vector of the magnetic mode in $m$-th layer; $\Delta \gamma_{m}$ is the $r$ and $q$ axes rotation at transition from $m$-th to $(m+1)$-th layer. This angle changes the sign at transition from one to another half of the crystal.

Determining the amplitude $A(\omega, H)$ of the wave (4) we could find dependences $\omega_{\text {res }}(H)$ by use of equation $\partial A / \partial \omega=0$. Theoretical fit of the experimental dependences $\omega_{\text {res }}(H)$ led to resonance curves represented in Fig. 1 (solid lines) [5]. We obtained the theoretical curves of two types that differ radically from the curves for pure nonmagnetic or magnetic modes corresponding to the case

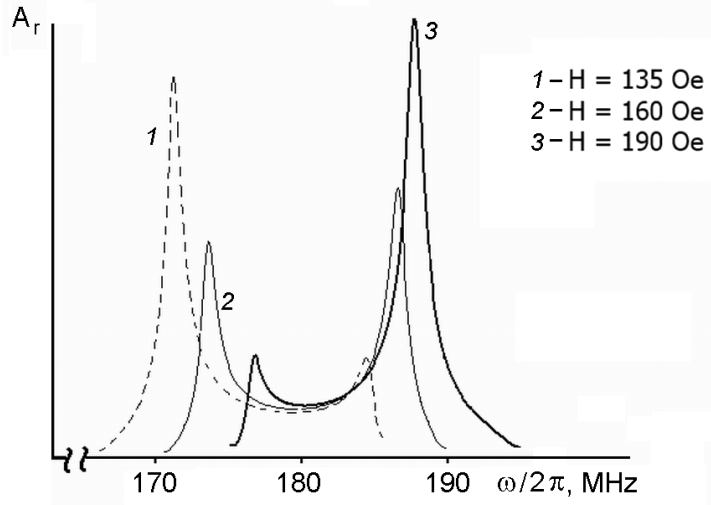

Fig. 4. Frequency dependence of amplitudes: the theory.

of uniform basal anisotropy (Fig. 1, dotted lines). This is a surprising result, which, however, correlates with the experiment [2]. Let us pay attention to several circumstances, demonstrated in Fig. 1. In the distance from the cross point of resonance curves for pure modes our curves are almost in coincidence with the corresponding curves for the pure modes. However not far from the cross point the principal differences appear. Values $\partial \omega / \partial H$ ("velocities" of resonances' shift) become nonzero for the both curves. One can see "interaction" of the curves, which appears in their mutual "repulsion" instead of crossing, as in the case of pure modes. Passing the cross point, the curve having described quasimagnetic resonances becomes corresponding to the quasinonmagnetic ones and vice versa.

To study behavior of the observed resonances (Fig. 2) we considered theoretical curves $A(\omega)$ at different fixed magnetic fields. Fig. 4 demonstrates the series of such curves, representing the frequency dependence of the traversed wave component $e_{r}^{\text {out }}$ (2) amplitude. One can see the "overflow" between the resonances like in the experiment (Fig. 2). This "overflow" appears in the following way. Amplitude of the left resonance decreases, while the amplitude of the right resonance increases. Simultaneously the "velocity" of the left resonance decreases, while the right one starts shifting ("passing the baton").

\section{Conclusion}

Qualitative agreement between the experimental (Fig. 2) and calculated (Fig. 4) resonance curves may be indicative of the validity of our basal-anisotropic nonuniform 
model and the nonuniform distribution of magnetization in the sample. It is important to note, that under electromagnetic excitation, in uniformly magnetized crystal magnetic modes should only be excited. Nonuniformity leads to appearance of the hybrid modes, as in the case of mechanical excitation [2].

Acknowledgements. This work was supported by the Grant No. F33.2/002 of State Fund for Fundamental Research of Ukraine, Russian Foundation for Basic Research, the Program of RAS "Acoustics of natural media".

\section{References}

1. M.H.Seavey, Sol.State Comm., 10, 219 (1972).

2. V.V.Tarakanov, V.I.Khizhniy, Low Temp. Phys., 22, 752 (1996).

3. Yu.N.Mitsay, K.M.Skibinsky, M.B.Strugatsky et al., JMMM, 219, 340 (2000).

4. M.B.Strugatsky, K.M.Skibinsky, V.V.Tarakanov, V.I.Khizhnyi, JMMM, 241, 330 (2002).

5. M.B.Strugatsky, K.M.Skibinsky, JMMM, 309, 64 (2007).

6. E.A.Turov, Zh.Eksp.Teor.Fiz., $\quad 96, \quad 2140$ (1989)

7. V.I.Ozhogin, V.L.Preobrazhenski, Zh.Eksp.Teor. -Fiz., 73, 988 (1977).

8. I.Sh.Akhmadullin, S.A.Migachev, M.F.Sadykov, M.M.Shakirzyanov, Fiz. Tverd. Tela, 47, 506 (2005) 